\title{
Creativity Illustration Presentation through Image Ways
}

\author{
Yu Jun \\ University of Shanghai for Science and Technology, Shanghai, China \\ jun_usst@163.com
}

Keywords: Image, Illustration presentation, Creativity thinking

\begin{abstract}
With the development of modern media, the applications of illustration already are not limited to the book illustrations. The forms and creating methods of illustration presentation are becoming more diversified; the illustrators' artistic accomplishments and creativities are becoming stronger. For today's illustrators, it is very important to find the proper ways to induce the re-creation ability of the reader. Through such ways, reader is no longer the passive accepter; he will become the primary former through his imagination when he confronts with the illustrations and the related texts. This paper analyzes how to make image expression in illustration creation, and the specific ways to the training of image creative thinking.
\end{abstract}

\section{Introduction}

Original illustration follows text to come into being. In China, the earliest illustration appeared in the presentations of sutra with the propagation of Buddhism, to enhance the infectivity and convincingness of text, which happened about 1300 years ago. The illustration with modern meanings was developed with the transitions of newspapers, periodicals and books, at the beginning of twenty century. Hence, the Chinese comprehensive dictionary gives the definition of illustration as: a picture attached to the book pages; printed in the body of text, or in the inserts, to be a supplementary explains to the text or to provide the effect of artistic appreciation [1]. With the quick development of information, the stage of text is no longer restricted in books, while illustration already enters into both paper and electronic publications, even become the prototypes of the creations of films, televisions and games. Besides servicing publications, illustration finds a more broad area in the development of commercial packing. Due to its interesting and vividness, illustration becomes the tightest combination of art and life. From early greeting cards to the modern T-shirt and amusing mug, illustration puts value added to the common commodity. At the same time, many kinds of derivatives of illustration have infiltrated into each parts of our lives.

So to say, modern illustration is far beyond the traditional meaning of "re-creating upon the base of literature". This paper describes how to enhance the creativity of illustration through image aesthetics, to give more imagine space to the production of illustration. Additionally, this paper discusses some available ways to help the originator to cultivate the creative thinking of image, which are important for the illustration artists.

\section{Enhance the Creativity of Illustration through Image Aesthetics}

The glossary of "Image" appeared in China long time ago. Zhouyi-Xici said that "extracting image from object" and "building image to explain the meaning". The "meaning" is the sublimation of the subject thought, while "image" gives the specific characterizing of the object. The "meaning" derives from ones heart inner and finds sustenance in the "image". So, image aesthetics is one character of the traditional Chinese culture. To accord with the theory of aesthetics, image can be represented in illustration through the following four catalogues

Expressing Abstractive Emotion through Specific Object Image. In this kind of image illustration, the specific picture may come from the real world. However, not the simple imitation of the real world, it is the visional mapping of the object world projected in the heart of the creator. The western aesthetics uses the word of "imitation image" to define it as: image created by subject through 
imitating the world of object; it is beyond the fact and the falsity; it is the representation image reflected in the feeling of subject; it is very likely to be similar as the object in the style and specific presentation; subject seems to back off. From the view of the illustration presentations, pictures of this kind of drawing are painted realistically. Most of them are presented through the methods of concrete describing, putting pictures together, and photographing. Through deconstruction re-combination, it presents the strain of thinking, as well as the conflict between the ideal and the reality. Its style has both the color of reality and the sense of stabilization, full of sparking of the thought. It purses not the truth of the life, but the reasonableness of vision. A representative illustrator is Leszek Zebrowski of Poland. His style is very special. Although his presentations come from those pictures of real faces and birds, they were full of visual impact and appeal, due to the construction of pictures and face expressions.

Expressing Deep Emotion through Artistic Subject Language. While "imitation image" emphasizes the picture of real object, "association image" gives more space for the creator. For those illustrations relying on the subject image, boundless hallucinations are visualized; the fictitious scenes that cannot exist in the real lives are presented, which make the abstractive thoughts and concepts to be presented in vision. Inspirited by emotion, aesthetic image makes up of one certain kind of atmosphere, temperament and situation, to transfer ones soul into the unlimited and indescribable perception region, through limited "image symbols". In such type of illustrations, instead of simply repeating or imitating the vision of the world, the author uses soul to feel the world, and then pass his feelings to the readers through artistic language.

Illustrators can use several artistic methods of reality, transformation, doodling and cartoon, as well as the artistic techniques like sound, light, electricity, color, smell, and flavors, to expatiate on flowery imagine and to build brilliant image. Its sculpt can be concise or complicated, its color can be lively or depressed, its style can be wispy or massive, its picture is to purse lifelikeness and interest, its meaning will be facetious and romantic. When used as book illustration, it always presents the deep understanding and apperceiving of the content of book for the illustrator. With some parts of the figures in the book, it gives a status that is non-existed in the real world, which touches the string in the heart. Japanese illustrator Kato Aya is good at making visual magic, with amazing imagination and gorgeous strange expression, constructing an dream world of strange things and enchanting charms. The viewer along the heart like and emotion left by Kato Aya, enters the path to the creations of their own. Folded in the mysterious dark atmosphere created by the visual features of these images, everyone can get the guide according to his own recognition of the world.

Personating or Anamorphosing Object Image through Borrowing Lyrics. Besides manufacturing the fantasy that does not exist in the reality, illustrator can also use the metaphor and association to make the common things in reality to be deformed, and then to present an image with special moral meaning. Due to the thing itself coming from reality, it is easier to trigger people thinking to obtain some certain synaesthesia. The creator takes the object image from the objective world and gives it some symbolic meanings, which in the western aesthetic consciousness is known as the "metaphor image". How to break the boundaries of a capacity between the mind and matter? The boundary between the mind and matter is the conscious characteristics of mind that can penetrate foreign objects to read the profound meaning. Only through painting skills, can illustrator articulate the intangible things in the mind.

Hence, Image generation and visualization is a very complex process, it needs to create a new form to provide a new perspective to explore the phenomenon, to explore the link between various phenomena, and the use of imagination is to unify, guide and inspire the formation of new thinking [2, 3]. Visual image is never emotional material for mechanical reproduction, but a creative grasp of reality, it is to grasp the image that is rich in imagination, creativity and acumen $[4,5]$. If imitation image is in accordance with the original look like a mirror image plane as to reflect the reality of life, that metaphor image is like a mirror image of three shuttles that go refraction life itself. Fig. 1 is Poland artist Stasys's "Wish you were here", which exhibits such effect. The image of a coffee cup becomes an accompanying deformation of wooden dolls of his childhood, is one of the series of 
wooden people created by the author. Hollow glazing round eyes of the wooden dolls convey complex information. A cup of coffee, together with a friend, it should be an enjoyable leisure time for the reminisced conversation. Faced with the sluggish empty expression of the dull, people are feeling the release of his melancholy memories and feelings of the author, and also to mobilize their own experiences and psychological impact. It is not so much an instant hit, as it was hit by his imagination and understanding [6].

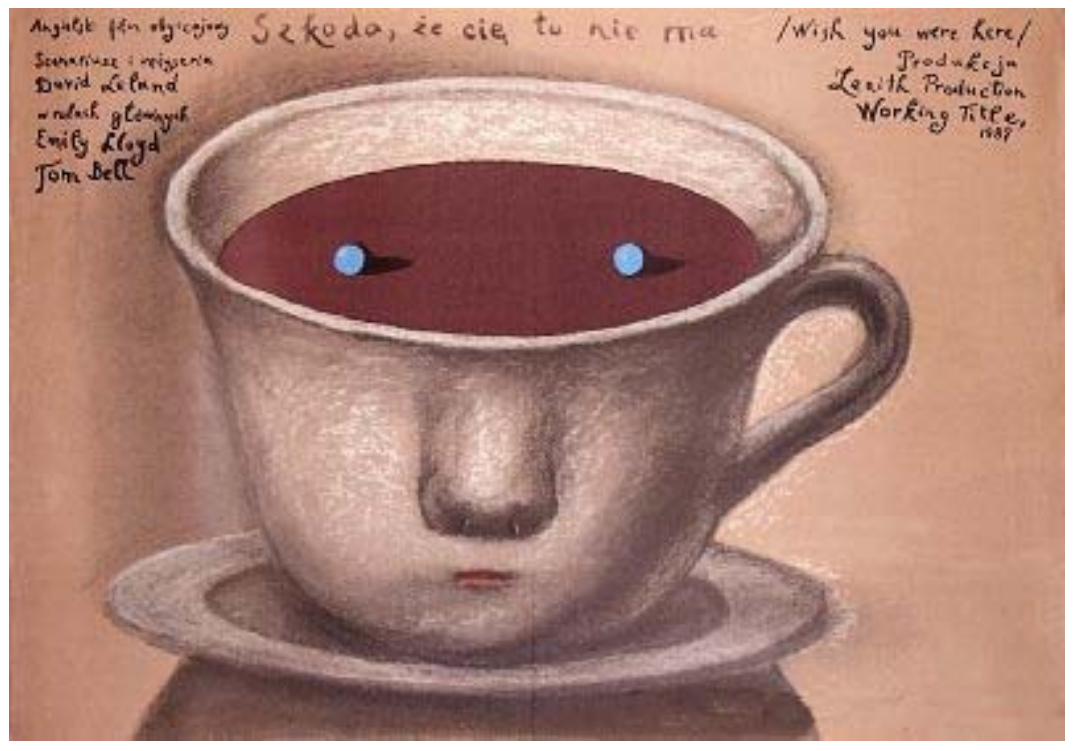

Figure 1. Illustration of Poland artist Stasys, " Wish you were here"

Presenting Abstractive Consciousness through Abnegating Specific Image. Abstracting is one kind of image to abandon depicting, using some pure form symbols to express emotion and awaken the viewers. The symbols used in art is a kind of metaphor, a kind of image containing open or hidden real meaning; art symbol is a kind of ultimate image - an irrational and expression not available in verbal image, resorting to direct perception of the image, filling of emotion, filling of life and personality, and resorting to the fell of living things. Therefore, it is also the birthplace of rational knowledge [7, 8, 9]. These characters of intent, ineffable, non-rational and emotional of artistic symbols just point to the knowledge of the human heart. The concrete and realistic presentation is very easy to limit people's thinking, while the abstract symbols is more likely to make people think freely, not rigidly adhere to see, and then produces resonance.

Compared with the text, illustrations can visualize the abstractive concept, transfer the specific communication content into the picture, so that the information is conveyed to be figurative language. It brings a different feel through its constructed image, so illustrator has a natural advantage of abstractive expressionism. The picture composition pattern, color contrast, symbol distribution all convey the mysterious emotion deviated from the object. An abstractive illustration can contain a large amount of information, waiting for the viewer to interpret. Like reading the ciphertext, through the form of pure signs to reach aesthetic perception, the audience eventually gets the emotional resonance. The particularity of many contemporary illustrations is distinguished as the authenticity of realism, illustrations in more of rejection of any idea of reality, it is more inclined to arouse our imagination and fantasy ...... contemporary illustration can be seen as an increasing platform to pursue abstractive ideas and personalized forms, it creates self needs, it makes the image have more broadness than precision, and more fantasy more than authenticity $[10,11]$. That is, humor, implicit and imaginative wisdom, arouses reader great emotion through incomparable thinking creativity.

\section{Culturing and Practicing Image Creative Creation and Thinking}

Image is to express abstractive emotion, so its creation will be slightly mysterious. To achieve image expression, one factor is to carry through the canvas with a brush, paper creation, technical 
learning tablet painting, and computer software processing; the other factor is to develop learners' creative thinking illustration, let them be creative and lively boldly, without departing from the subject expression. That is, free moving between images and charm is the focus of training creative illustrator. This makes the thinking of illustrator to be more creative with independent initiative, this also requires illustrator having sensitive awareness of the living environment, extensions of a variety of painting in the form, and ability of the initiative associating.

Breaking Life Experience. Creativity is often lost because they are limited to the inertia of thinking, experience accumulated in life is to destroy the creativity. Be good at breaking the common graphics and awareness of life, try to use the association and imagination wings to make creation. Illustrator creates some productions that can not be found in the real world with rich experience. So. viewer should guide themselves through the metaphor of the screen, open the senses to perceive the diversity of nature and life's complexity, expand thinking activities, thereby inducing a creative force. The image of the screen viewer can develop thinking, stimulate the imagination of its potential, from one image to create other ones, reverse thinking; then, the ambiguity of the pictures can activate the viewer to enter into a broad thinking space. Thus, each person produces personalized psychological feelings.

Carrier and Technique Innovation. Different from ordinary art painting, illustrator performance was implemented on a specific carrier. Book illustrations are printed on publications, become parts of books; commercial illustrations are the combination of the posters, packaging, advertising materials, with more abundant carrier materials. Figure 2 "Summer Etude illustrator" is one example of such diversity. In the same part of the original illustration, it is decorated with color badge, while the badge is printed with the same illustration. In such way, graphic and three-dimensional combinations enrich illustrator level and sensitivity, develop a series of derivatives.

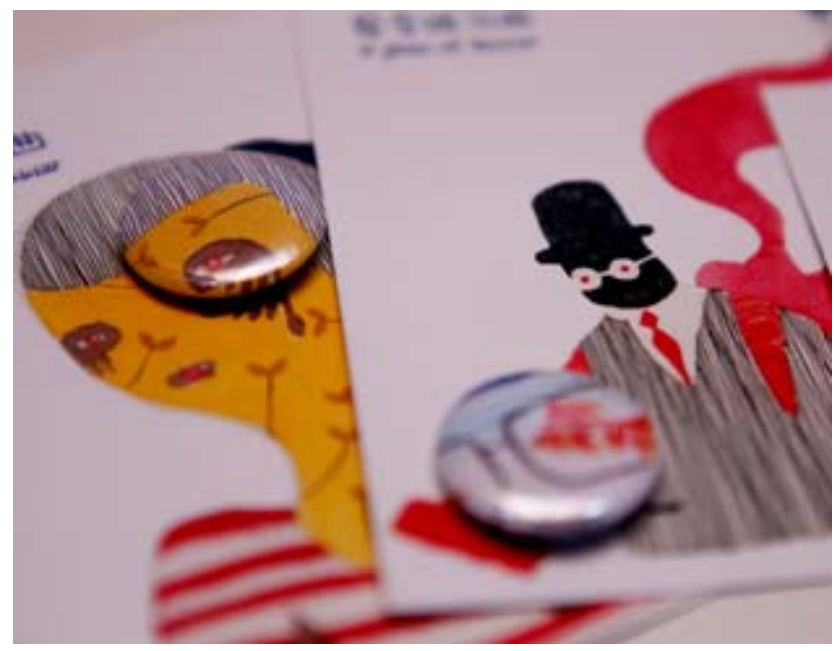

Figure 2. Illustration of Summer Etude, Hejie, China

Considering creation techniques, modern illustrator creation software provides illustrators unprecedented convenience. Using Photoshop software can achieve architecture and restructuring of the images, illustrators will not spend much cost on the composition, color up, and repeated attempts to pursue the best visual effects. Combined with hand-painted plates, illustrator can become more invincible and more creative. AI illustrator software provides simple plugs such as filter, which can quickly achieve fantastic results screen. Hand-painted artwork, watercolor washes and painting strokes, are all very helpful to complete the combined effect and have been widely adopted by illustrators worldwide. However, technology is not the sticking point, technological innovation must service the innovative thinking.

Examples of Image Expression Thinking Training. In practical illustration image thinking training, some unique, unusual and even subversive methods can realize fine training effect. For example, the main characters, times, places, actions, scenes, etc., can be broken up, and then 
reassembled as a creative material. Fig. 3 is such an example based on a fantastic topic of "A jubilant yangko dancing fish in the road”. Through restricting the material of the illustration creation, but also to try to figure out the character in the context, it is to realize the expression of surrealism content. Even originated from a normal proposition, it can also through the screen to subvert the common color, composition to subvert the sequence of time and space, special or use texture, collage, integrated media to achieve the special effects, so as to make the reality beyond the "image" expression to achieve the best results.

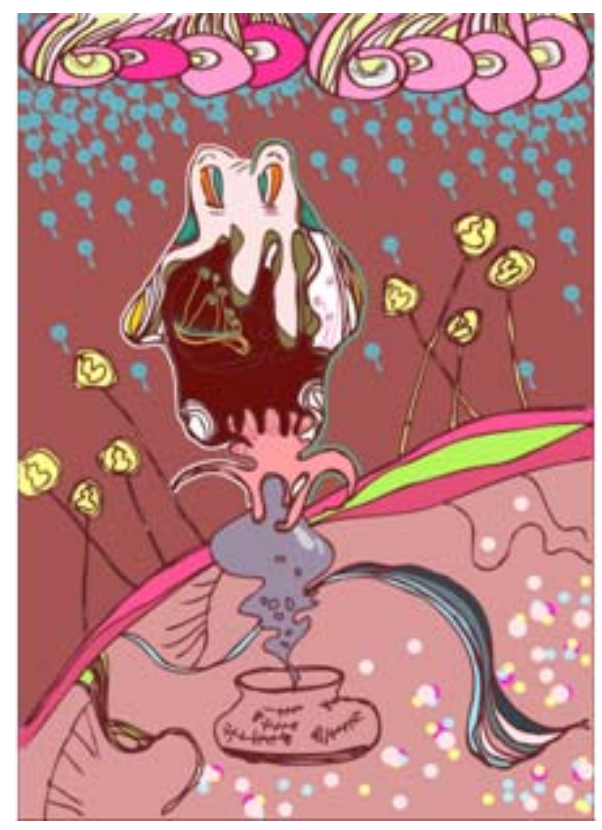

Figure 3. Illustration of a jubilant yangko dancing fish in the road, Hemin, China

Another form of training is based on grouping. Group members do not communicate with each others about the content of the creation in advance. The same piece of paper is in turn to be handled by each group number. According to some abstractive painting traces left by a formers, the succeeding members extend it further, until reaching the last one. Synthesizing the works left on paper, the presentation is finalized by hand-drawn or computer processing. Based on the backgrounds, symbols and other information, subsequent members make imagination, association or connection. In such way, image thinking can be trained; at the same time, the ability of finalization can be practiced.

\section{Conclusions}

Image illustration expression is driven by the illustrator's subjective emotion and achieved by skilled painting techniques; it is the expression of the abstractive elements into personal perception, temperament, and culture of the human spirit. Compared with the common illustration, image illustration is full of creator's emotion and consciousness, which gives it distinctive personal style and features. For the viewers living in the same cultural environment, excellent image illustrations will cause a strong aesthetic resonance, while the viewers who are not familiar with their culture can also enrich the visual and psychological experience, to achieve the effect of emotional expression. Creating image illustration is a challenge to the imagination and creativity of the illustrator; related works often give viewers fresh and new viewing experiences beyond realistic experiences. In short, more creative image ways can efficiently enrich the illustration presentations.

\section{References}

[1] Cihai, Shanghai lexicographic publishing house, Shanghai, China (1999)

[2] Pasi Valiaho, Futures of Images, Oxford Art, v37, n1 (2014) 
[3] Lijianwen, Image design illustration, Huazhong University of Science and Technology press, Wuhan, China (2006)

[4] Rudolf Arne Heim, Art and visual perception, Chinese Social Science Press, Beijing (1984)

[5] Hanneke Grootenboer, The Pensive Image: On Thought in Jan van Huysum's Still Life Paintings, Oxford Art, v34, n1 (2011)

[6] Roanne Bell, Mark Sinclair, Pictures and Words: New Comic Art and Narrative Illustration, Yale University Press (2003)

[7] Susan Langer, Issues of Art, Chinese Social Science Press, Beijing (1983)

[8] Karen T. Taylor, Forensic Art and Illustration, CRC Press LLC (2001)

[9] Catherine Fowler, Obscurity and Stillness: Potentiality in the Moving Image, Art Journal, v72, n1 (2013)

[10] Thomas E Wartenberg, Beyond Mere Illustration: How Films Can Be Philosophy, The Journal of Aesthetics and Art Criticism, v64, n1 (2006)

[11] Zhaochenyin, Illustration art course, Zhejiang people's Fine Arts Publishing House, China (2009) 